\title{
ESTRUCTURA Y COMPOSICIÓN FLORÍSTICA DE UN BOSQUE HÚMEDO TROPICAL DEL PARQUE NACIONAL NATURAL CATATUMBO BARÍ, COLOMBIA
}

Palabras clave: Andes, bosque húmedo tropical, Catatumbo, Colombia, composición florística, flora neotropical. Key words: Andes, tropical wet forest, Catatumbo, Colombia, floristic composition, neotropical flora.

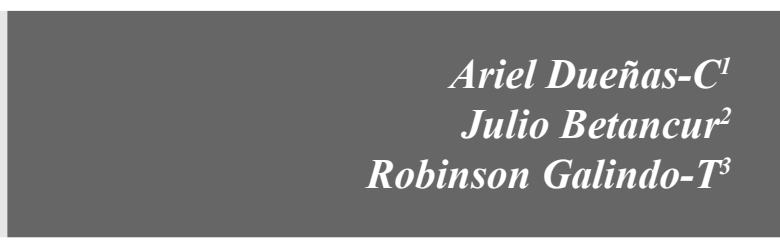

su orden, Arecaceae, Rubiaceae, Burseraceae, Lauraceae y Moraceae. Se puede decir que este es un bosque relativamente heterogéneo con una baja riqueza de especies, como resultado de la degradación en el pasado. Cordillera Oriental colombiana, departamento Norte de Santander, ubicado a $700 \mathrm{~m}$ de altitud. En 0.1 ha se censaron todos los individuos con $\mathrm{DAP} \geq 1 \mathrm{~cm}$. Se encontraron 636 individuos y 109 especies con DAP $\geq 1 \mathrm{~cm}$ y 432 individuos y 90 especies con DAP $\geq 2.5 \mathrm{~cm}$. Las familias con más especies fueron Lauraceae (13), Rubiaceae (10), Melastomataceae (9) y Arecaceae (6), mientras que los géneros con más especies fueron Ocotea y Miconia (7), Psychotria (5) y Eschweilera, Inga y Piper (4). La mayor parte de los individuos y de las especies se presentaron en los intervalos inferiores de altura y de clases diamétricas. El área basal total fue de $5.65 \mathrm{~m}^{2}$. La especies de mayor importancia ecológica en la comunidad (mayor IVI) fueron, en su orden, Trattinnickia cf. burserifolia, Calathea inocephala, Brownea ariza, Oenocarpus minor y Euterpe precatoria. Las familias más importantes (mayor IVF) fueron, en

\begin{abstract}
The floristic composition and structure of a tropical wet forest in the Natural National Park Catatumbo Barí were characterized. This natural park is located in the Cordillera Oriental of Colombia, Norte de Santander, at 700 m altitude. All individuals with $\mathrm{dbh} \geq 1 \mathrm{~cm}$ found in a 0.1 ha plot were sampled. We have found 636 individuals and 109 species with $\mathrm{dbh} \geq 1 \mathrm{~cm}$, and 432 individuals and 90 species with dbh $\geq 2.5 \mathrm{~cm}$. The families with the highest number of species were Lauraceae (13), Rubiaceae (10), Melastomataceae (9), and Arecaceae (6). The genera with the higher number of species were Ocotea and Miconia (7), Psychotria (5), and Eschweilera, Inga and Piper (4). The majority of individuals and species corresponded
\end{abstract}

Escuela de Biología, Facultad de Ciencias, Universidad Industrial de Santander, Apartado 678, Bucaramanga, Colombia. jadc1991012@tux. uis.edu.co

2 Instituto de Ciencias Naturales, Universidad Nacional de Colombia, Apartado 7495, Bogotá, Colombia. jcbetancurb@.unal.edu.co

3 Regional Norandina, Parques Nacionales Naturales de Colombia, Ministerio de Ambiente, Vivienda y Desarrollo Territorial, Av. Quebrada Seca No.30-44, Bucaramanga, Colombia.rgtgalindo@gmail.com 
to lowest height range and diametric class. The total basal area was $5.65 \mathrm{~m}$. The most ecologically important species (highest IVI) were, in order, Trattinnickia cf. burserifolia, Calathea inocephala, Brownea ariza, Oenocarpus minor and Euterpe predatoria, and the families were Arecaceae, Rubiaceae, Burseraceae, Lauraceae and Moraceae. This is a relatively heterogeneous forest with low species richness, as a result of its past degradation.

\section{INTRODUCCIÓN}

La región tropical de Suramérica presenta gran variedad de tipos de vegetación boscosa, que comprenden desde las regiones de tierras bajas hasta las de alta montaña, y desde las pluviales hasta las áridas (Huber \& Riina 1997). Sin embargo, gran parte de su superficie está ocupada por lo que se denomina como bosque húmedo tropical (bh-T) y las sabanas de tierras bajas (Van der Hammen 1992). Los bosques húmedos tropicales se caracterizan por ser ecosistemas con gran complejidad estructural y ambiental, además de que albergan la mayor diversidad de especies de plantas del mundo, concentrando cerca del $50 \%$ de las especies descritas (Gentry 1993).

La extensión del bh-T en Colombia es de $415.000 \mathrm{~km}^{2}$ (Etter 1993) lo cual equivale al $36.5 \%$ del territorio nacional. Este tipo de bosque se distribuye a través de las principales regiones biogeográficas del país, como lo son las tierras bajas del Pacífico o Chocó Biogeográfico (con cerca de 4.6000.000 ha) la Amazonía y algunos sectores de la Orinoquia (con cerca de $36.400 .000 \mathrm{ha})$ y las estribaciones de los Andes, en los valles medios de los ríos Magdalena y Sinú, en los valles bajos de los ríos Cauca y San Jorge y en la cuenca del río Catatumbo (con cerca de 1.650.000 ha) (IAvH 1997).

El Parque Nacional Natural Catatumbo Barí fue creado con el fin de proteger el último re- licto de bosque húmedo tropical existente en el extremo nororiental del país, así como por albergar algunos asentamientos de la comunidad indígena Barí, una de las más amenazadas en nuestro territorio (Castaño-Uribe \& Cano 1998). Los bosques de la cuenca del río Catatumbo han sido degradados por cerca del $65 \%$ de su cobertura original, pues de una extensión original de aproximadamente 550.000 ha sólo se conservan unas 200.000 ha (Etter 1993). Esta región de Colombia ha sido incluida por varios autores dentro de la denominada Provincia de Maracaibo (Morrone 2001) o del Refugio del Catatumbo (Hernández-C. et al. 1992).

Algunos autores han señalado la importancia de los bosques del Catatumbo, definiéndolos como selvas húmedas de piso térmico cálido con la presencia de algunas especies de plantas y animales que sugieren afinidades con las biotas chocoana, centroamericana, amazónica y del valle medio del río Magdalena (Hernández-C. et al. 1992). Sin embargo, se conoce muy poco sobre esta región de Colombia, especialmente sobre su vegetación, por lo cual este trabajo explora la estructura y la composición florística de un bosque húmedo tropical ubicado allí.

\section{ÁREA DE ESTUDIO}

El estudio se realizó en el Parque Nacional Natural Catatumbo Barí, el cual hace parte del Sistema de Parques Nacionales Naturales de la República de Colombia. Este parque tiene una extensión de 158.125 ha, se encuentra ubicado en el extremo nororiental del departamento de Norte de Santander y comprende parte de la cuenca del río Catatumbo, el cual confluye al lago de Maracaibo en Venezuela.

El muestreo se realizó en el cerro Brubusquera, situado en cercanías de la comunidad de Bridicayra (etnia Barí), vereda Santa Fe de

Revista Colombia Forestal Vol. 10 No. 20 - Diciembre 2007 
Honduras, corregimiento de Honduras Motilonía, municipio de Convención. Al sitio de muestreo se accede por la carretera que conduce desde la ciudad de Convención hacia el corregimiento de Cartagenita, para posteriormente acceder a la comunidad Bridicayra por camino de herradura.

El cerro Brubusquera presenta pendientes inferiores al $30 \%$, está ubicado aproximadamente a $700 \mathrm{~m}$ de altitud, $8^{\circ} 54^{\prime}$ de latitud Norte y $73^{\circ} 15^{\prime}$ de longitud Oeste. La estación pluviométrica más cercana es Hacharira, ubicada a $75 \mathrm{~m}$ de altitud, para la cual se tiene una precipitación promedio anual de $4726 \mathrm{~mm}$, con un periodo de lluvias entre octubre y noviembre (ca. $640 \mathrm{~mm}$ en promedio) y un periodo seco entre enero y marzo (130-220 $\mathrm{mm}$ en promedio). El cerro permanece la mayor parte del año cubierto de niebla, especialmente en las horas de la mañana, y está dentro de la zona de vida del bosque húmedo tropical (bh-T) (Espinal-T. 1977).

\section{MÉTODOS}

\section{MUESTREO}

El muestreo se realizó entre marzo y noviembre de 2005. La elección del sitio de muestreo se hizo mediante el uso de cartografía aérea, teniendo en cuenta que tuviera una cobertura boscosa homogénea y estuviera alejado de claros o senderos. Se siguió la metodología de inventarios rápidos propuesta por Gentry (1995) y las modificaciones sugeridas por otros autores (Franco-Rosselli et al. 1997, Marín-Corba \& Betancur 1997, Mendoza-C. 1999, GalindoT. et al. 2003). Se establecieron diez transectos de $50 \times 2$ m cada uno ( 0.1 ha en total) que se orientaron aleatoriamente, teniendo en cuenta que no se traslaparan. Dentro de cada transecto se censaron y recolectaron todos los individuos con diámetro a la altura del pecho (DAP) $\geq 1 \mathrm{~cm}$, a cada uno de los cuales se le registró la altura (estimada visualmente) y el perímetro o circunferencia a la altura del pecho (CAP) aproximadamente $1.3 \mathrm{~m}$ por encima del suelo. Los individuos ubicados en el límite del transecto se incluyeron sólo si al menos la mitad de su tronco se encontraba dentro del área demarcada. En el caso de plantas con tallos múltiples o que se ramifican por debajo de $1.3 \mathrm{~m}$ de altura o de palmetos (herbáceas acaules), cada uno de los brotes, ramas o pecíolos se midieron independientemente.

\section{PROCESAMIENTO DE LA INFORMACIÓN}

Los especímenes testigos fueron depositados en los herbarios COL y UIS. El CAP se transformó a DAP según la ecuación DAP = CAP/ $\pi$. En el caso de plantas acaules o ramificadas por debajo de la altura del pecho, el DAP total (Dt) se calculó según lo propuesto por FrancoRosselli et al. (1997), así: $\mathrm{D} t=(4 \mathrm{~A} t / \pi)^{1 / 2}$, en donde $\mathrm{A} t=\sum \mathrm{A} i, \mathrm{~A} i=\pi(\mathrm{DAP})^{1 / 2}, \mathrm{~A} t=$ área total y $\mathrm{A} i=$ área de cada brote. Posteriormente, los DAP se transformaron a área basal a través de la ecuación $\mathrm{AB}=\pi / 4(\mathrm{DAP})^{2}$ (MuellerDombois \& Ellenberg 1974).

Se calculó el índice de valor de importancia (IVI) para cada especie como la sumatoria de la densidad (DeR), la frecuencia (FR) y la dominancia (DoR) relativas (Finol 1976), en donde DeR $=(\#$ de individuos de la especie/\# total de individuos en la comunidad) x 100; FR $=$ (\# de sub transectos en los que aparece la especie/sumatoria de las frecuencias de todas las especies $) \times 100 ;$ DoR $=(\Sigma \mathrm{AB}$ de todos los individuos de la especie $/ \Sigma \mathrm{AB}$ de toda la comunidad) x 100. Para evaluar la distribución de cada una de las variables ecológicas estudiadas se construyeron intervalos de clase mediante la ecuación $\mathrm{C}=(\mathrm{Xmáx}$. $\mathrm{Xmin}.) / \mathrm{m}$, donde $\mathrm{C}=$ amplitud del intervalo; $\mathrm{m}=1+3.3 \log \mathrm{N} ; \mathrm{N}=$ No. de individuos (Rangel-Ch. \& Velásquez 1997). 
También se calculó el índice de valor de importancia de las familias (IVF), como la sumatoria de la densidad, la dominancia y la riqueza relativas de cada familia, según lo propuesto por Mori \& Boom (1983).

\section{RESULTADOS}

\section{RIQUEZA FLORÍSTICA}

Se encontraron 636 individuos con DAP $\geq 1$ $\mathrm{cm}$, distribuidos en 109 especies, 77 géneros y 49 familias. Así mismo, se encontraron 90 especies con DAP $>2.5 \mathrm{~cm}$, discriminadas en 73 especies y 329 individuos con DAP entre $2.5 \mathrm{~cm}$ y $10 \mathrm{~cm}$ y 46 especies y 103 individuos con DAP $\geq 10 \mathrm{~cm}$. Las familias con mayor número de especies fueron Lauraceae con trece, Rubiaceae con diez, Melastomataceae con nueve, Arecaceae con seis y Euphorbiaceae, Lecythidaceae, Mimosaceae y Moraceae con cinco especies cada una. Por otra parte, los géneros con más especies fueron Ocotea (Laura- ceae) y Miconia (Melastomataceae) con siete, Psychotria (Rubiaceae) con cinco e Inga (Mimosaceae), Eschweilera (Lecythidaceae) y Piper (Piperaceae) con cuatro especies cada uno (Anexo 1).

\section{ESTRUCTURA VERTICAL DEL BOSQUE}

La distribución vertical de los individuos muestra que la mayor parte de ellos (52\%) tienen alturas entre 1.3 y $4.9 \mathrm{~m}$, punto a partir del cual la disminución en altura se hace progresiva. Por otra parte, sólo nueve individuos $(1.4 \%)$ presentaron alturas superiores a 23.5 m (Figura 1).

La distribución de las especies según la altura de los individuos tuvo un comportamiento similar a la mostrada por los individuos totales, con la mayor parte de ellas en los intervalos inferiores de altura. De hecho, en el primer intervalo de altura (1.3-4.9 m) están representadas el $63 \%$ de las especies totales, punto a partir

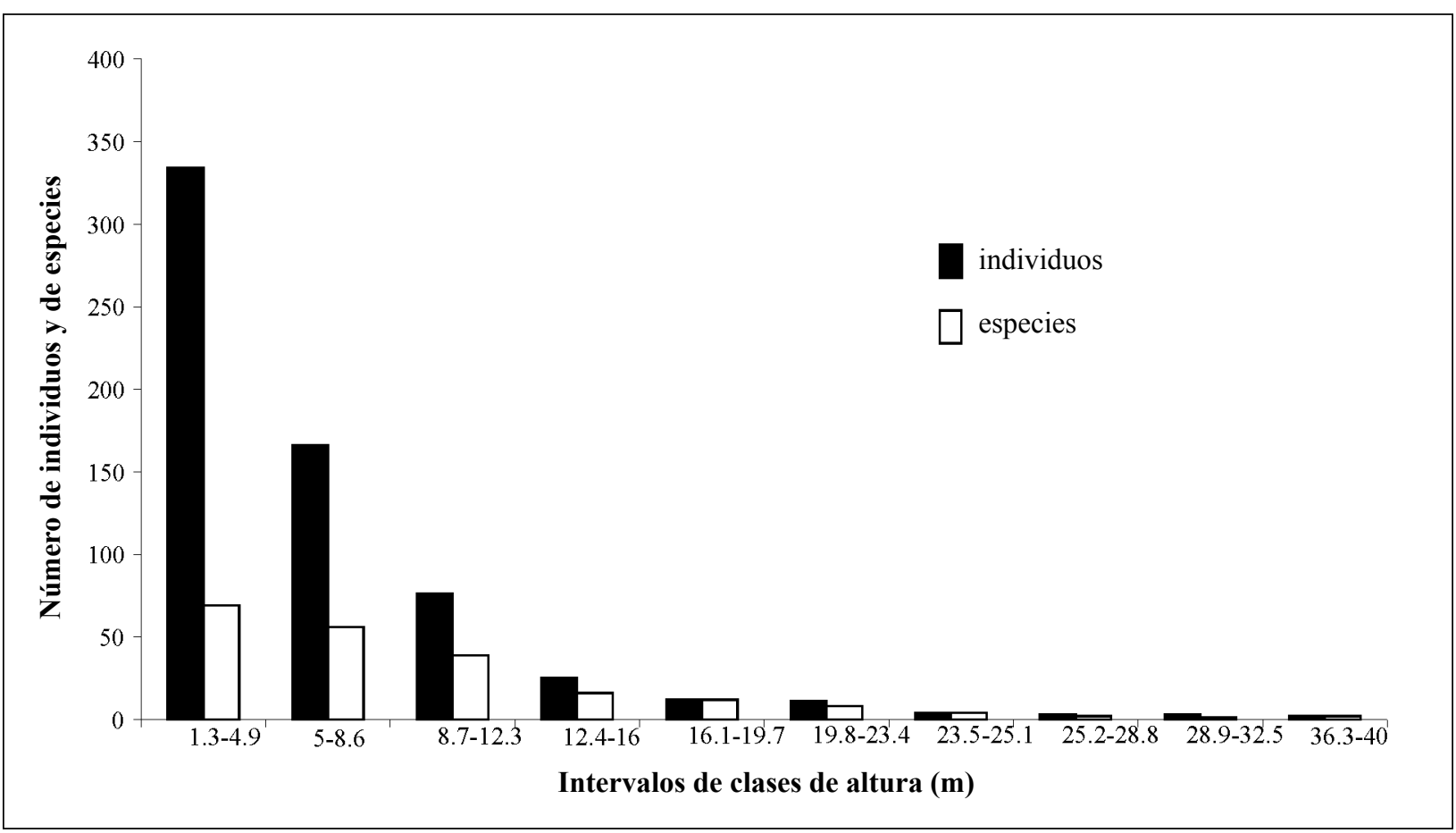

Figura 1. Distribución vertical de especies e individuos con DAP $\geq 2.5 \mathrm{~cm}$.en 0.1 ha de un bosque húmedo tropical del PNN Catatumbo Barí. 
del cual hay una disminución progresiva de las mismas (Figura 1).

El área basal total acumulada por la comunidad fue de $5.65 \mathrm{~m}^{2}$ (Anexo 1). La mayor parte de los individuos estuvieron en los intervalos de clases diamétricas menores (entre 1.1 y $10.4 \mathrm{~cm}$ de DAP), punto a partir del cual la disminución en el número de individuos fue drástica (Figura 2).

\section{VALOR DE IMPORTANCIA DE LAS ESPECIES (IVI)}

Las especies más abundantes en el bosque fueron la marantácea Calathea inocephala y las palmas Euterpe precatoria, Geonoma sp., Oenocarpus minor y Wettinia praemorsa, las cuales aportaron el $31.6 \%$ de los individuos totales. Así mismo, las especies con mayor dominancia relativa fueron, en su mismo orden, Trattinnickia cf. burserifolia (Burseraceae), Brownea ariza (Caesalpiniaceae), Sloanea guianensis (Elaeocarpaceae), Erythrina poeppigiana ( $\mathrm{Fa}-$ baceae) y Ficus dugandii (Moraceae), las cuales acumularon el 50\% del área basal de la comunidad (Tabla 1).

Tabla 1. Especies con mayor valor de importancia (IVI), densidad y dominancia relativas en un bosque húmedo tropical del PNN Catatumbo Barí.

\begin{tabular}{|l|l|r|}
\hline Parámetros ecológicos & \multicolumn{1}{|c|}{ Especies } & Valor \\
\hline Densidad relativa (\%) & Euterpe precatoria & 8.0 \\
& Oenocarpus minor & 7.5 \\
& Calathea inocephala & 6.9 \\
& Wettinia praemorsa & 5.0 \\
& Geonoma sp. & 4.2 \\
Dominancia relativa (\%) & Trattinnickia & \\
& cf. burserifolia & 18.7 \\
& Brownea ariza & 10.2 \\
& Sloanea guianensis & 8.2 \\
& Erythrina poeppigiana & 6.8 \\
& Ficus dugandii & 6.1 \\
Índice de valor de & Trattinnickia & \\
importancia (IVI/300) & cf. burserifolia & 23.5 \\
& Calathea inocephala & 15.1 \\
& Brownea ariza & 14.8 \\
& Oenocarpus minor & 13.7 \\
& Euterpe precatoria & 13.2 \\
\hline
\end{tabular}

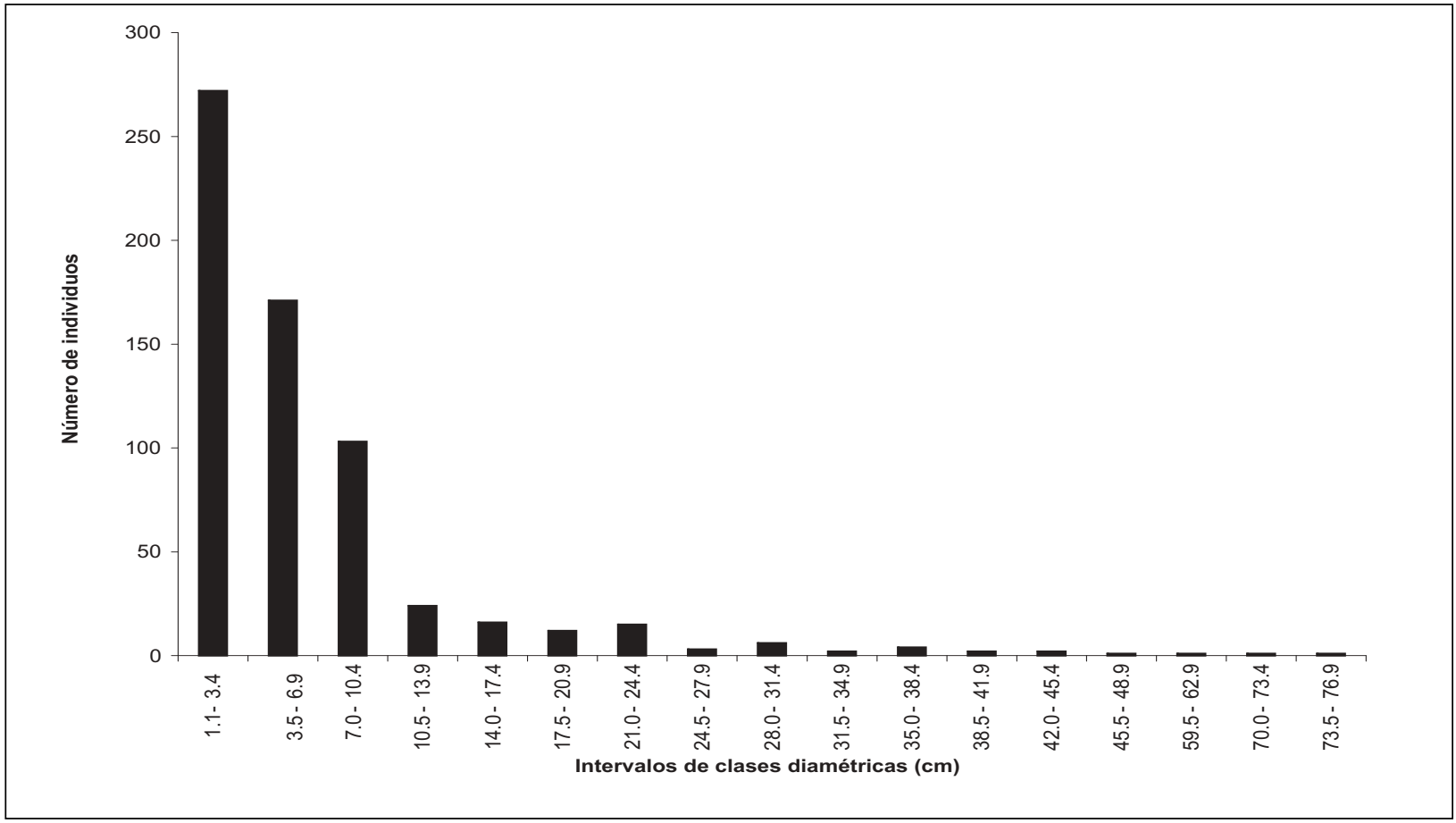

Figura 2. Distribución de los individuos con $\mathrm{DAP}^{\circ} 2.5 \mathrm{~cm}$ en 0.1 ha de acuerdo a las clases diamétricas, en un bosque húmedo tropical del PNN Catatumbo Barí. 
Las especies con más importancia ecológica en la comunidad, en su mismo orden, fueron Trattinnickia cf. burserifolia (Burseraceae), Calathea inocephala (Marantaceae), Brownea ariza (Caesalpiniaceae) y las palmas Oenocarpus minor y Euterpe precatoria. Las cinco especies anteriores acumularon el $26.8 \%$ del IVI total de la comunidad (Tabla 1).

\section{VALOR DE IMPORTANCIA DE LAS FAMILIAS (IVF)}

La familia que presentó más individuos fue Arecaceae, con poco más de la cuarta parte de los individuos totales, seguida de Rubiaceae (con el $11 \%$ de los individuos), Marantaceae (7.2\%) y Lauraceae (6.3\%). Las familias que acumularon más área basal fueron Burseraceae, con casi una quinta parte del total, seguida de Caesalpiniaceae (con el 10.4\% del área basal total) Arecaceae $(8.3 \%)$ y Elaeocarpaceae $(8.2 \%)$ (Tabla 2 , Anexo 1). La familia con más importancia ecológica en la comunidad vegetal fue Arecaceae (40.8/300), seguida de Rubiaceae, Burseraceae y Lauraceae, las tres últimas con valores bastante similares (25.9-24.5/300) (Tabla 2).

Tabla 2. Familias de plantas ecológicamente más importantes en un bosque húmedo tropical del PNN Catatumbo Barí.

\begin{tabular}{|c|c|c|c|c|c|}
\hline Familias & 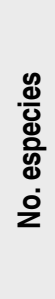 & 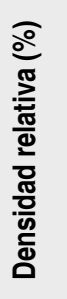 & 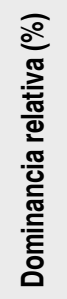 & 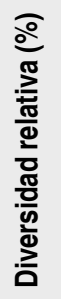 & $\begin{array}{l}\text { 올 } \\
\frac{\text { }}{\underline{1}}\end{array}$ \\
\hline Arecaceae & 6 & 27.0 & 8.3 & 5.5 & 40.8 \\
\hline Rubiaceae & 10 & 11.0 & 5.7 & 9.2 & 25.9 \\
\hline Burseraceae & 3 & 3.6 & 18.8 & 2.8 & 25.1 \\
\hline Lauraceae & 13 & 6.3 & 6.3 & 11.9 & 24.5 \\
\hline Moraceae & 5 & 4.9 & 7.7 & 4.6 & 17.1 \\
\hline Melastomataceae & 9 & 5.0 & 2.4 & 8.3 & 15.7 \\
\hline Caesalpiniaceae & 1 & 3.1 & 10.4 & 0.9 & 14.5 \\
\hline Marantaceae & 2 & 7.2 & 5.2 & 1.8 & 14.3 \\
\hline
\end{tabular}

\section{DISCUSIÓN}

\section{RIQUEZA FLORÍSTICA}

A nivel de familias, la composición florística del bosque del Catatumbo estudiado es similar a la registrada para otros bosques húmedos andinos ubicados a altitudes similares (Phillips \& Miller 2002).

En la Tabla 3 se presentan datos sobre la riqueza de especies de algunos bosques andinos situados a altitudes similares a la del bosque estudiado (entre 400 y $1180 \mathrm{~m}$ ) y obtenidos con métodos similares (muestreos de individuos con DAP $\geq 2.5 \mathrm{~cm}$ en $0.1 \mathrm{ha}$ ). Se observa que la riqueza encontrada en el bosque del Catatumbo es característicamente menor si se compara con la registrada para otros bosques andinos muy húmedos, como los de Murrí en Colombia, Centinela, Huamaní, Jatun Sacha y Miazi en Ecuador y La Genoa, Río Candamo y Río Távara en Perú. Sin embargo, es muy superior a la registrada para otros bosques andinos situados al sur del continente americano (Argentina, Bolivia y Chile) o similar a la registrada para otros bosques mucho menos lluviosos, como los de Alto del Mirador y Mariquita en Colombia o San Sebastián en Ecuador (Tabla 3, Phillips \& Miller 2002).

Los resultados obtenidos en este estudio están de acuerdo con la afirmación de Hernández-C. et al. (1992) respecto a que la biota del Catatumbo comparte muchos elementos con las del Chocó biogeográfico, la cuenca amazónica y el valle medio del río Magdalena, al menos teniendo en cuanta los elementos florísticos de mayor importancia en el bosque (Rentería 1977, Cortés et al. 1998, Balcázar et al. 2000).

\section{ESTRUCTURA}

Uno de los aspectos estructurales más notorios en el bosque estudiado, y en general del Catatumbo, es la alta densidad de palmas. De he-

Revista Colombia Forestal Vol. 10 No. 20 - Diciembre 2007 
Tabla 3. Riqueza florística de bosques situados entre 400 y 1150 m de altitud, para muestras de 0.1 ha e individuos con DAP $\geq 2.5 \mathrm{~cm}$.

\begin{tabular}{|c|c|c|c|c|c|}
\hline $\begin{array}{c}\text { Localidades } \\
\text { (datos de Gentry, } \\
\text { en Phillips } \\
\text { \& Miller 2002) }\end{array}$ & 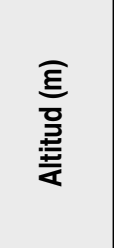 & 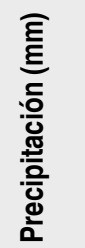 & 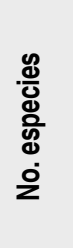 & 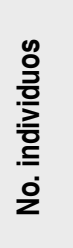 & 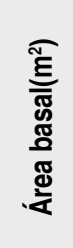 \\
\hline Tarapoto, Perú & $350-540$ & 1400 & 100 & 471 & 2.57 \\
\hline Río Távara, Perú & 400 & 6760 & 200 & 381 & 4.73 \\
\hline $\begin{array}{l}\text { Jatun Sacha, } \\
\text { Ecuador }\end{array}$ & 450 & 4100 & 240 & 435 & 4.55 \\
\hline $\begin{array}{l}\text { Perro Muerto, } \\
\text { Ecuador }\end{array}$ & 450 & 1550 & 69 & 285 & 3.18 \\
\hline Peyehue, Chile & $460-520$ & 3000 & 16 & 336 & 24.81 \\
\hline $\begin{array}{l}\text { San Sebastian, } \\
\text { Ecuador }\end{array}$ & 550 & 2000 & 96 & 420 & 4.66 \\
\hline Mariquita, Colombia & 560 & 1920 & 94 & 337 & 5.25 \\
\hline Centinela, Ecuador & $650-800$ & 4500 & 140 & 411 & 3.95 \\
\hline $\begin{array}{l}\text { Catatumbo, } \\
\text { Colombia } \\
\text { (este estudio) }\end{array}$ & 700 & 4726 & 90 & 432 & 5.65 \\
\hline Rio Candamo, Perú & 790 & 6760 & 236 & 445 & 5.09 \\
\hline $\begin{array}{l}\text { Alto de Mirador, } \\
\text { Chile }\end{array}$ & 800 & 4000 & 16 & 334 & 8.71 \\
\hline $\begin{array}{l}\text { Cerros de Amotape, } \\
\text { Perú }\end{array}$ & 820 & & 54 & 395 & 3.45 \\
\hline Miazi, Ecuador & 850 & 2000 & 186 & 416 & 5.01 \\
\hline Murrí, Colombia & 910 & 4200 & 179 & 327 & 4.4 \\
\hline $\begin{array}{l}\text { Parque El Rey, } \\
\text { Argentina }\end{array}$ & 1000 & 1500 & 41 & 190 & 3.34 \\
\hline $\begin{array}{l}\text { Chaquimayo, } \\
\text { Bolivia }\end{array}$ & 1000 & 1600 & 81 & 463 & 4.71 \\
\hline $\begin{array}{l}\text { Alto de Mirador, } \\
\text { Colombia }\end{array}$ & 1180 & 2500 & 89 & 320 & 5.23 \\
\hline La Genoa, Perú & 1140 & 2000 & 112 & 345 & 3.77 \\
\hline Huamaní, Ecuador & 1150 & 3500 & 156 & 402 & 5.13 \\
\hline
\end{tabular}

cho, tres de las cinco especies con mayor valor de densidad relativa correspondieron a palmas (Tabla 1). Otros autores han registrado resultados similares para varios sitios y regiones de Colombia, como en bosques andinos premontanos (Franco-Rosselli et al. 1997) o bosques bajos del Chocó biogeográfico (Galeano et al.
1998) aunque en este último caso se registraron sólo individuos con DAP $\geq 10 \mathrm{~cm}$. Similarmente, Gentry (1986) afirmaba que la alta densidad de palmas es una característica fisonómica de los bosques húmedos tropicales. En algunos sectores del bosque estudiado las palmas cubrían áreas extensas, restringiendo fuertemente la entrada de la luz y el crecimiento de otras especies en el sotobosque.

De acuerdo con los resultados obtenidos sobre la distribución vertical y por clases diamétricas de los individuos y de las especies (Figuras 1,2) junto con lo observado en el campo, podríamos afirmar que el bosque estudiado tiene cuatro estratos principales, así: 1) el estrato inferior o sotobosque, en el cual son muy abundantes Calathea inocephala y Psychotria bertieroides y que llega aproximadamente a 5 $\mathrm{m}$ de altura. C. inocephala en algunos sectores del bosque crecía formando densas agregaciones de vástagos, especialmente en áreas con algún tipo de disturbio, como los ocasionados por los frecuentes deslizamientos de tierra y la caída de árboles; 2) el estrato intermedio, en el cual son muy abundantes las palmas Euterpe precatoria y Oenocarpus minor y que alcanza aproximadamente hasta $12 \mathrm{~m}$ de altura, siendo este estrato el que acumula la mayor área basal dentro de la comunidad. Entre el sotobosque y el estrato medio es muy abundante Brosimum alicastrum; 3) el dosel o estrato superior, el cual puede alcanzar aproximadamente hasta 24 $m$ de altura y es bastante heterogéneo en cuanto a la composición de especies, pero en el que domina Trattinnickia $\mathrm{cf}$. burserifolia; 4) el estrato emergente, el cual está conformado por árboles muy espaciados entre sí, que pueden alcanzar hasta $38 \mathrm{~m}$ de altura y que no logran formar un continuo en la parte superior del bosque; hay predominio de Erythrina poeppigiana, Sloanea guianensis y Trattinnickia $\mathrm{cf}$. burserifolia.

El área basal registrada en 0.1 ha para el bosque del Catatumbo $\left(5.65 \mathrm{~m}^{2}\right)$ es superior a la 
conocida para cualquier otro bosque andino del noroccidente de Suramérica. Sin embargo, es inferior a la registrada para los bosques australes de Alto del Mirador y Peyehue en Chile (Tabla 3). Cabe observar que los altos valores de área basal acumulada sólo por unas pocas especies influyeron notoriamente en la importancia ecológica de ellas dentro de la comunidad. Así, sólo cinco especies acumularon el $50 \%$ de la dominancia total de la comunidad (Tabla 1). Las primeras posiciones en la dominancia relativa ocupadas por Trattinnickia cf. burserifolia, Brownea ariza y Sloanea guianensis se puede explicar teniendo en cuenta que sólo quince individuos de estas especies tenían más de $20 \mathrm{~cm}$ de DAP. Similarmente, Erythrina poeppigiana y Ficus dugandii, con solo 4 individuos cada uno, tuvieron diámetros entre 20 y $60 \mathrm{~cm}$ (Anexo 1).

También es importante resaltar que este bosque es relativamente heterogéneo, pues la importancia ecológica de las especies que lo componen está repartida más homogéneamente que en otros bosques andinos (Gentry 1995, Franco-Rosselli et al. 1997, Galindo-T. et al. 2003). De hecho, las primeras cinco especies más importantes acumularon sólo cerca de una cuarta parte del IVI total de la comunidad (Tabla 1).

La modificación a la metodología de inventarios rápidos propuesta por Gentry (1995), en cuanto a la inclusión de individuos con DAP entre 1 y $2.5 \mathrm{~cm}$, ocasionó un incremento en 204 individuos y 20 especies. Evidentemente, con esta modificación se incrementa ostensiblemente la información sobre la composición y la estructura de los estratos inferiores del bosque, los que generalmente no se consideran en este tipo de trabajos. Resultados similares han sido divulgados por otros autores, como Franco-Rosselli et al. (1997) para bosques andinos premontanos, Mendoza-C. (1999) para bosques secos de tierras bajas y Galindo-T. et al. (2003) para bosques andinos y altoandinos.
En general, los resultados obtenidos y la comparación de los mismos con los de otros bosques andinos con similar altitud y precipitación (Tabla 3) indican que el bosque estudiado tiene una riqueza baja que ha sido objeto de algún tipo de intervención antrópica, la cual se manifiesta en la extracción selectiva de maderas y de los árboles con mayor porte, así como en la condición generalizada en la región relacionada con la tala del bosque para el establecimiento de cultivos ilícitos.

\section{AGRADECIMIENTOS}

Al Instituto de Ciencias Naturales de la Universidad Nacional de Colombia, a la Escuela de Biología de la Universidad Industrial de Santander y a la Dirección Territorial Norandina del Sistema de Parques Nacionales Naturales, por las facilidades proporcionadas para el desarrollo de esta investigación. A los funcionarios A. Cortés, H.Valderrama y J. Martínez del Parque Nacional Natural Catatumbo Barí, por su colaboración y acompañamiento en el campo. A la comunidad indígena de Bridicayra por su hospitalidad. A los botánicos que ayudaron en la determinación de los especimenes: R. Bernal (Arecaceae), R. Callejas (Piperaceae), Z. Cordero (Melastomataceae), J. L. Fernández (varios grupos), O. Rivera (varios grupos), J. C. Murillo (Annonaceae, Euphorbiaceae, Pteridophyta), C. Romero (Mimosaceae), L. K. Ruiz (Leguminosae s.1.), N. Salinas (Costaceae), S. Suárez (Marantaceae) y W. Vargas (Lauraceae).

\section{REFERENCIAS BIBLIOGRÁFICAS}

Balcazar-Vargas, M. P., J. O. Rangel-CH. \& E. L. Linares-C. 2000. Diversidad florística de la Serranía de Las Quinchas, Magdalena medio (Colombia). Caldasia 22(2): 191-224.

Castaño-Uribe, C. \& M. Cano. 1998. El Sistema de Parques Nacionales Naturales de Co- 
lombia. Unidad Administrativa Especial del Sistema de Parques Nacionales Naturales, Ministerio del Medio Ambiente de Colombia. Editorial Nomos. Bogotá, Colombia.

Cortés-B., R., P. Franco-Rosselli \& J. O. Rangel-CH. 1998. La flora vascular de la Sierra de Chiribiquete, Colombia. Caldasia 20(2): 103-141.

Espinal-T., L. S. 1977. Zonas de Vida o Formaciones Vegetales de Colombia. Memoria explicativa sobre el mapa ecológico de Colombia. Instituto Geográfico Agustín Codazzi. pg. Bogotá, Colombia.

Etter, A. 1993. Diversidad Ecosistemica en Colombia Hoy. pgs. en: S. Cárdenas \& H. D. Correa (eds.). Nuestra Diversidad Biológica. Colección Maria Restrepo de Ángel \& CEREC, Fundación Alejandro Escobar. Bogotá, Colombia.

Finol, H. 1976. Estudio fitosociológico de las unidades 2 y 3 de la Reserva Forestal de Carapo, Estado de Barinas. Acta Botánica Venezuelica 10 (1-4): 15-103.

Franco-Roselli, P., J. Betancur \& J. L. Fernández-Alonso. 1997. Diversidad florística en dos bosques subandinos del sur de Colombia. Caldasia 19(1-2): 205-234.

Galeano, G., J. Cediel \& M. Pardo. 1998. Structure and Floristic Composition of a One Hectare Plot of Wet Forest at the Pacific Coast of Chocó, Colombia. Chapter 28. pgs. en: F. Dalmeier \& J. Comiskey (eds.). Forest Biodiversity in North, Central and South America, and the Caribbean: Research and Monitoring. Man and the Biosphere Series. Parthenon Publishing. Washington D.C., U. S. A.

Galindo-T., R., J. Betancur, J. J. Cadena-M. 2003. Estructura y composición florística de cuatro bosques andinos del Santuario de Flora y Fauna Guanentá-Alto Río Fonce, Cordillera Oriental colombiana. Caldasia 25(2): 313-335.

Gentry, A. H. 1986. Species richness and floristic composition of Chocó region plant communities. Caldasia 15 (71-75): 71-91.

Gentry, A. H. 1993. El Significado de la Biodiversidad. pgs. en: S. Cárdenas \& H. D. Correa (eds.), Nuestra diversidad Biológica. Colección María Restrepo de Angel \& CEREC, Fundación Alejandro Escobar. Bogotá, Colombia.

Gentry, A. H. 1995. Patterns of Diversity and Floristic Composition in Neotropical Montane Forests. pgs. 103-126 en: S. P. Churchill, H. Balslev, E. Forero \& J. L. Luteyn (eds.). Biodiversity and Conservation of Neotropical Montane forests. The New York Botanical Garden. New York, U. S. A.

Hernández-C., J., A. Hurtado, R. Ortiz. \& T. Walschburger. 1992. Unidades Biogeográficas de Colombia. pgs. 150-173 en G. Halffter (comp.). La Diversidad Biológica de Iberoamérica I. Acta Zoológica Mexicana, Volumen especial.

Huber, O. \& R. Riina (eds.). 1997. Glosario Fitoecológico de las Américas. Vol. 1 América del Sur: Países Hispanoparlantes. Ediciones Tamandúa. 500 pg. Caracas, Venezuela.

IAVH (Instituto de Investigación de Recursos Biológicos Alexander Von Humboldt). 1997. Informe Nacional Sobre el Estado de la Biodiversidad en Colombia. Tres volúmenes. Instituto de Investigación de Recursos Biológicos Alexander von Humboldt \& PNUMA, Ministerio del Medio Ambiente. Bogotá, Colombia. 
Marín-Corba, C. A. \& J. Betancur. 1997. Estudio florístico en un robledal del Santuario de Flora y Fauna de Iguaque (Boyacá, Colombia). Revista Acad. Colomb. Cienc. 21 (80): 249-259.

Mendoza-C., H. 1999. Estructura y riqueza florística del bosque seco tropical en la región Caribe y el valle del río Magdalena, Colombia. Caldasia 21 (1): 70-94.

Mori, S. \& B. Boom. 1983. Ecological importance of Myrtaceae in a Eastern Brazilian forest. Biotropica 15 (1): 68-70.

Morrone, J .J. 2001. Biogeografía de América Latina y el Caribe. M. \& T. Manuales y Tesis SEA.Vol. 3. pg. Zaragoza, México.

Mueller-Dombois, D. \& H. Ellenberg. 1974. Aims and Methods of Vegetation Ecology. John Wiley \& Sons, Inc. 547 pg. New York, U. S. A.
Phillips, O. \& J. S. Miller. 2002. Global patterns of plant diversity: Alwyn H. Gentry's forests transect data set. Monographs in Systematic Botany from the Missouri Botanical Garden Vol: 89: 164-198.

Rangel-CH., J. O. \& A P. Velázquez. 1997. Métodos de Estudio de la Vegetación. pgs. 59-88 en: J. O. Rangel-Ch., P. D. Lowy-C. \& M. Aguilar-P. (eds.). Colombia Diversidad Biótica II, Tipos de Vegetación en Colombia. Instituto de Ciencias Naturales, Universidad Nacional de Colombia. Bogotá, Colombia.

Renteria, E. 1977. Contribución al estudio de la flora de Santander del Sur. Actualidades Biológicas 6 (21): 70-79.

Van Der Hammen, T. 1992. Historia, Ecología y Vegetación. Corporación Colombiana para la Amazonia, "Araracuara" (COA). 411 pg. Bogotá, Colombia. 
Anexo 1. Especies registradas en 0.1 ha en un bosque húmedo tropical del PNN Catatumbo Barí (el número de colección corresponde a la serie de numeración de Dueñas-Cepeda).

\begin{tabular}{|c|c|c|c|c|c|c|c|c|c|c|c|}
\hline 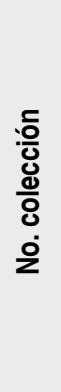 & Familias y especies & 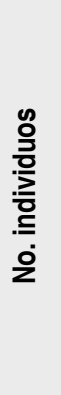 & 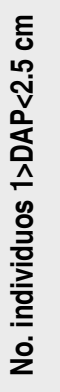 & 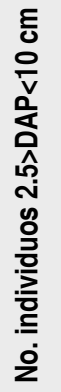 & 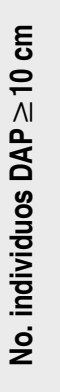 & 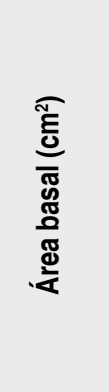 & 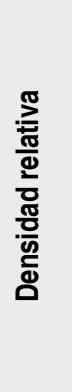 & 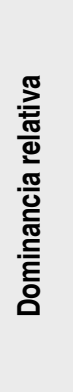 & 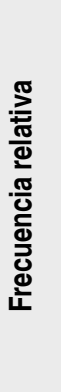 & 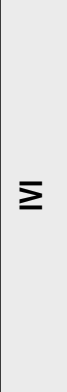 & 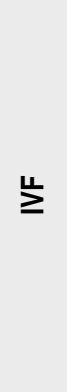 \\
\hline $\begin{array}{l}806 \\
891\end{array}$ & $\begin{array}{l}\text { ANACARDIACEAE } \\
\text { Astronium graveolens Jacq. } \\
\text { Tapirira guianensis Aubl. }\end{array}$ & $\begin{array}{l}1 \\
1\end{array}$ & 1 & 1 & & $\begin{array}{l}1.2 \\
1.3\end{array}$ & $\begin{array}{l}0.2 \\
0.2\end{array}$ & $\begin{array}{l}0.0 \\
0.0\end{array}$ & $\begin{array}{l}0.4 \\
0.5\end{array}$ & $\begin{array}{l}0.6 \\
0.7\end{array}$ & 2.2 \\
\hline 895 & $\begin{array}{l}\text { ANNONACEAE } \\
\text { Guatteria platyphylla Triana \& Planch. }\end{array}$ & 1 & 1 & & & 1.6 & 0.2 & 0.0 & 0.5 & 0.7 & 1.2 \\
\hline 873 & $\begin{array}{l}\text { APOCYNACEAE } \\
\text { Bonafousia sananho (Ruíz \& Pav.) Markgr. }\end{array}$ & 1 & & 1 & & 24.8 & 0.2 & 0.0 & 0.5 & 0.7 & 1.1 \\
\hline 951 & $\begin{array}{l}\text { AQUIFOLIACEAE } \\
\text { Ilex aff. fructiclipeata Cuatrec. }\end{array}$ & 1 & & & 1 & 412.5 & 0.2 & 0.7 & 0.5 & 1.0 & 1.9 \\
\hline $\begin{array}{l}836 \\
778 \\
755 \\
816 \\
879 \\
983\end{array}$ & $\begin{array}{l}\text { ARECACEAE } \\
\text { Bactris cf. pilosa Karst. } \\
\text { Chamaedorea pinnatifrons Oerst. } \\
\text { Euterpe precatoria Mart. } \\
\text { Geonoma sp. } \\
\text { Oenocarpus minor Mart. } \\
\text { Wettinia praemorsa (Willdenow) J.G.W. Boer }\end{array}$ & $\begin{array}{r}12 \\
1 \\
51 \\
27 \\
48 \\
32\end{array}$ & $\begin{array}{l}1 \\
8 \\
9 \\
8 \\
6\end{array}$ & $\begin{array}{l}39 \\
18 \\
34 \\
26\end{array}$ & $\begin{array}{l}4 \\
6\end{array}$ & $\begin{array}{r}359.2 \\
1.0 \\
1530.2 \\
330.1 \\
1780.7 \\
693.9\end{array}$ & $\begin{array}{l}1.9 \\
0.2 \\
8.0 \\
4.2 \\
7.0 \\
5.0\end{array}$ & $\begin{array}{l}0.6 \\
0.0 \\
2.7 \\
0.6 \\
3.1 \\
1.2\end{array}$ & $\begin{array}{l}1.0 \\
0.5 \\
2.5 \\
3.9 \\
2.8 \\
1.5\end{array}$ & $\begin{array}{r}3.5 \\
0.7 \\
13.2 \\
9.0 \\
13 \\
7.7\end{array}$ & 40.8 \\
\hline 979 & $\begin{array}{l}\text { BIGNONIACEAE } \\
\text { Jacaranda copaia D. Don }\end{array}$ & 5 & & 5 & & 125.8 & 0.8 & 0.2 & 1.0 & 2.0 & 1.9 \\
\hline 829 & $\begin{array}{l}\text { BORAGINACEAE } \\
\text { Cordia bicolor A. DC. }\end{array}$ & 1 & & & 1 & 424.1 & 0.2 & 0.8 & 0.5 & 1.4 & 1.9 \\
\hline $\begin{array}{l}915 \\
885 \\
899\end{array}$ & $\begin{array}{l}\text { BURSERACEAE } \\
\text { Protium heptaphyllum March. } \\
\text { Trattinnickia cf. aspera (Standl.) Sw. } \\
\text { Trattinnickia cf. burserifolia Mart. }\end{array}$ & $\begin{array}{r}1 \\
4 \\
18\end{array}$ & $\begin{array}{l}3 \\
1\end{array}$ & $\begin{array}{l}1 \\
1 \\
8\end{array}$ & 9 & $\begin{array}{r}5.7 \\
16.0 \\
10572.8\end{array}$ & $\begin{array}{l}0.2 \\
0.6 \\
2.8\end{array}$ & $\begin{array}{r}0.0 \\
0.0 \\
18.7\end{array}$ & $\begin{array}{l}0.5 \\
0.5 \\
2.0\end{array}$ & $\begin{array}{r}0.7 \\
1.1 \\
23.5\end{array}$ & 25.1 \\
\hline 358 & $\begin{array}{l}\text { CAESALPINIACEAE } \\
\text { Brownea ariza Benth. }\end{array}$ & 20 & 5 & 10 & 5 & 5735.7 & 3.1 & 10.2 & 1.5 & 14.8 & 14.5 \\
\hline 856 & $\begin{array}{l}\text { CAPPARIDACEAE } \\
\text { Capparis macrophylla Kunth }\end{array}$ & 7 & 4 & 3 & & 77.0 & 1.0 & 0.1 & 1.5 & 2.7 & 2.2 \\
\hline 884 & $\begin{array}{l}\text { CECROPIACEAE } \\
\text { Cecropia peltata L. }\end{array}$ & 4 & 2 & 1 & 1 & 103.04 & 0.6 & 1.8 & 1.0 & 3.5 & 3.5 \\
\hline 944 & $\begin{array}{l}\text { CELASTRACEAE } \\
\text { Perrottetia calva Cuatrec. }\end{array}$ & 1 & & 1 & & 40.3 & 0.2 & 0.1 & 0.5 & 0.7 & 1.1 \\
\hline
\end{tabular}


Ariel Dueñas-C. / Julio Betancur / Robinson Galindo-T.

\begin{tabular}{|c|c|c|c|c|c|c|c|c|c|c|c|}
\hline 883 & $\begin{array}{l}\text { CHLORANTHACEAE } \\
\text { Hedyosmum racemosum (Ruíz \& Pav.) G. Don }\end{array}$ & 1 & & 1 & & 11.5 & 0.2 & 0.0 & 0.5 & 0.7 & 1.1 \\
\hline 924 & $\begin{array}{l}\text { CHRYSOBALANACEAE } \\
\text { Licania parvifructa Fanshawe \& Maguire }\end{array}$ & 1 & 1 & & & 1.6 & 0.2 & 0.0 & 0.5 & 0.7 & 1.1 \\
\hline 952 & $\begin{array}{l}\text { CLUSIACEAE } \\
\text { Clusia cf. columnaris Engl. }\end{array}$ & 1 & & & 1 & 249.6 & 0.2 & 0.4 & 0.5 & 1.0 & 1.7 \\
\hline $\begin{array}{l}822 \\
739\end{array}$ & $\begin{array}{l}\text { COSTACEAE } \\
\text { Costus pulverulentus C. Presl } \\
\text { Costus sp. }\end{array}$ & $\begin{array}{l}3 \\
2\end{array}$ & $\begin{array}{l}3 \\
2\end{array}$ & & & $\begin{array}{l}3.5 \\
2.0\end{array}$ & $\begin{array}{l}0.5 \\
0.3\end{array}$ & $\begin{array}{l}0.0 \\
0.0\end{array}$ & $\begin{array}{l}0.5 \\
0.5\end{array}$ & $\begin{array}{l}1.0 \\
0.8\end{array}$ & 2.6 \\
\hline 960 & $\begin{array}{l}\text { CYATHEACEAE } \\
\text { Cyathea nigripens (C.Chr.) Domin }\end{array}$ & 10 & 1 & 9 & & 293.2 & 1.6 & 0.5 & 2.0 & 4.1 & 3.0 \\
\hline 788 & $\begin{array}{l}\text { CYCLANTACEAE } \\
\text { Carludovica palmata Ruíz \& Pav. }\end{array}$ & 3 & & 2 & 1 & 158.1 & 0.5 & 0.3 & 1.0 & 1.7 & 1.7 \\
\hline 905 & $\begin{array}{l}\text { ELAEOCARPACEAE } \\
\text { Sloanea guianensis Benth. }\end{array}$ & 15 & 1 & 8 & 6 & 4632.3 & 2.4 & 8.2 & 1.5 & 12.0 & 11.7 \\
\hline $\begin{array}{l}775 \\
906 \\
750 \\
923 \\
913\end{array}$ & $\begin{array}{l}\text { EUPHORBIACEAE } \\
\text { Acalypha diversifolia Jacq. } \\
\text { Alchornea integrifolia Pax \& K. Hoffm. } \\
\text { Hieronyma alchorneoides Allemão var. stipulosa P. Franco } \\
\text { Hieronyma oblonga (Tul.) Müell.Arg. } \\
\text { Maprounea guianensis Aubl. }\end{array}$ & $\begin{array}{l}3 \\
3 \\
1 \\
1 \\
1\end{array}$ & $\begin{array}{l}1 \\
1\end{array}$ & $\begin{array}{l}3 \\
1 \\
1\end{array}$ & 1 & $\begin{array}{r}43.8 \\
977.4 \\
43.9 \\
2.0 \\
1.6\end{array}$ & $\begin{array}{l}0.5 \\
0.5 \\
0.2 \\
0.2 \\
0.2\end{array}$ & $\begin{array}{l}0.1 \\
1.7 \\
0.1 \\
0.0 \\
0.0\end{array}$ & $\begin{array}{l}0.5 \\
1.5 \\
0.5 \\
0.5 \\
0.5\end{array}$ & $\begin{array}{l}1.0 \\
3.7 \\
0.7 \\
0.7 \\
0.7\end{array}$ & 7.9 \\
\hline 703 & $\begin{array}{l}\text { FABACEAE } \\
\text { Erythrina poeppigiana O.F. Cook }\end{array}$ & 4 & & & 4 & 3819.5 & 0.6 & 6.8 & 1.5 & 8.9 & 8.5 \\
\hline 867 & $\begin{array}{l}\text { FLACOURTIACEAE } \\
\text { Casearia commersoniana Cambess. }\end{array}$ & 5 & 2 & 2 & 1 & 678.3 & 0.8 & 1.2 & 1.0 & 3.0 & 2.9 \\
\hline 743 & $\begin{array}{l}\text { ICACINACEAE } \\
\text { Citronella aff. incarum (J.F. Macbr.) R.A. Howard }\end{array}$ & 1 & 1 & & & 2.0 & 0.2 & 0.0 & 0.5 & 0.7 & 1.2 \\
\hline $\begin{array}{l}869 \\
959 \\
864 \\
751 \\
765 \\
897 \\
757 \\
914 \\
862 \\
937 \\
793 \\
963 \\
853\end{array}$ & $\begin{array}{l}\text { LAURACEAE } \\
\text { Aniba coto (Rusby) Kosterm. } \\
\text { Aniba perutilis Hemsl. } \\
\text { Aniba puchury-minor Mez } \\
\text { Nectandra sp. } 1 \\
\text { Nectandra sp. } 2 \\
\text { Ocotea guianensis Aubl. } \\
\text { Ocotea macrophylla Kunth } \\
\text { Ocotea sp. } 1 \\
\text { Ocotea sp. } 2 \\
\text { Ocotea sp. } 3 \\
\text { Ocotea sp. } 4 \\
\text { Ocotea sp. } 5 \\
\text { Indeterminada }\end{array}$ & $\begin{array}{r}1 \\
1 \\
1 \\
1 \\
2 \\
22 \\
3 \\
1 \\
2 \\
2 \\
1 \\
2 \\
1\end{array}$ & $\begin{array}{r}12 \\
1\end{array}$ & $\begin{array}{l}1 \\
1 \\
2 \\
7 \\
2 \\
1 \\
2\end{array}$ & 2 & $\begin{array}{r}137.1 \\
35.1 \\
86.7 \\
28.8 \\
23.0 \\
1830.1 \\
19.2 \\
23.0 \\
757.5 \\
87.0 \\
86.7 \\
36.1 \\
401.2\end{array}$ & $\begin{array}{l}0.2 \\
0.2 \\
0.2 \\
0.2 \\
0.3 \\
3.5 \\
0.6 \\
0.2 \\
0.3 \\
0.3 \\
0.2 \\
0.3 \\
0.2\end{array}$ & $\begin{array}{l}0.2 \\
0.1 \\
0.2 \\
0.1 \\
0.0 \\
3.2 \\
0.0 \\
0.0 \\
1.3 \\
0.2 \\
0.2 \\
0.1 \\
0.7\end{array}$ & $\begin{array}{l}0.5 \\
0.5 \\
0.5 \\
0.5 \\
0.5 \\
1.5 \\
0.9 \\
0.5 \\
0.5 \\
0.5 \\
0.5 \\
0.5 \\
0.0\end{array}$ & $\begin{array}{l}0.9 \\
0.7 \\
0.8 \\
0.7 \\
0.8 \\
8.2 \\
1.6 \\
0.7 \\
2.1 \\
1.0 \\
0.8 \\
0.9 \\
1.4\end{array}$ & 24.5 \\
\hline $\begin{array}{l}934 \\
870 \\
930\end{array}$ & $\begin{array}{l}\text { LECYTHIDACEAE } \\
\text { Eschweilera albiflora Miers } \\
\text { Eschweilera andina (Rusby) J.F. Macbr. } \\
\text { Eschweilera cf. antioquensis Dugand \& Daniel }\end{array}$ & $\begin{array}{l}1 \\
1 \\
1\end{array}$ & $\begin{array}{l}1 \\
1\end{array}$ & 1 & & $\begin{array}{l}5.7 \\
1.6 \\
1.0\end{array}$ & $\begin{array}{l}0.2 \\
0.2 \\
0.2\end{array}$ & $\begin{array}{l}0.0 \\
0.0 \\
0.0\end{array}$ & $\begin{array}{l}0.5 \\
0.5 \\
0.5\end{array}$ & $\begin{array}{l}0.7 \\
0.7 \\
0.7\end{array}$ & 5.2 \\
\hline
\end{tabular}




\begin{tabular}{|c|c|c|c|c|c|c|c|c|c|c|c|}
\hline $\begin{array}{l}966 \\
825\end{array}$ & $\begin{array}{l}\text { Eschweilera aff. sclerophylla Cuatrec. } \\
\text { Gustavia cf. hexapetala Sm. }\end{array}$ & $\begin{array}{l}1 \\
1\end{array}$ & & $\begin{array}{l}1 \\
1\end{array}$ & & $\begin{array}{r}8.0 \\
35.1\end{array}$ & $\begin{array}{l}0.2 \\
0.2\end{array}$ & $\begin{array}{l}0.0 \\
0.1\end{array}$ & $\begin{array}{l}0.5 \\
0.5\end{array}$ & $\begin{array}{l}0.7 \\
0.7\end{array}$ & \\
\hline $\begin{array}{l}769 \\
921\end{array}$ & $\begin{array}{l}\text { MARANTACEAE } \\
\text { Calathea inocephala (Kuntze) H.Kenn. \& Nicolson } \\
\text { Ischnosiphon arouma (Aubl.) Körn. }\end{array}$ & $\begin{array}{r}44 \\
2\end{array}$ & $\begin{array}{l}3 \\
2\end{array}$ & 30 & 11 & $\begin{array}{r}2936.5 \\
6.9\end{array}$ & $\begin{array}{l}6.9 \\
0.3\end{array}$ & $\begin{array}{l}5.2 \\
0.0\end{array}$ & $\begin{array}{l}3.0 \\
0.5\end{array}$ & $\begin{array}{r}15.0 \\
0.8\end{array}$ & 14.3 \\
\hline $\begin{array}{l}842 \\
949 \\
810 \\
909 \\
904 \\
852 \\
980 \\
926 \\
813\end{array}$ & $\begin{array}{l}\text { MELASTOMATACEAE } \\
\text { Bellucia pentamera Naudin } \\
\text { Graffenrieda cf. conostegioides Triana } \\
\text { Miconia cf. affinis DC. } \\
\text { Miconia aponeura Triana } \\
\text { Miconia Iongifolia (Aubl.) DC. } \\
\text { Miconia nervosa Triana } \\
\text { Miconia pterocaulon Triana } \\
\text { Miconia punctata (Desr.) D.Don } \\
\text { Miconia cf. serrulata Naudin }\end{array}$ & $\begin{array}{l}1 \\
6 \\
3 \\
7 \\
3 \\
2 \\
7 \\
7 \\
3\end{array}$ & $\begin{array}{l}1 \\
1 \\
1 \\
1 \\
4 \\
5 \\
2\end{array}$ & $\begin{array}{l}5 \\
1 \\
5 \\
2 \\
1 \\
2 \\
1 \\
1\end{array}$ & $\begin{array}{l}1 \\
1 \\
1\end{array}$ & $\begin{array}{r}147.1 \\
145.3 \\
176.0 \\
279.5 \\
134.4 \\
69.8 \\
291.6 \\
330.7 \\
52.6\end{array}$ & $\begin{array}{l}0.2 \\
0.9 \\
0.5 \\
1.1 \\
0.4 \\
0.3 \\
1.1 \\
1.1 \\
0.5\end{array}$ & $\begin{array}{l}0.3 \\
0.3 \\
0.3 \\
0.5 \\
0.2 \\
0.1 \\
0.5 \\
0.6 \\
0.1\end{array}$ & $\begin{array}{l}0.5 \\
1.0 \\
0.5 \\
1.0 \\
0.9 \\
0.5 \\
1.5 \\
0.5 \\
1.0\end{array}$ & $\begin{array}{l}0.9 \\
2.0 \\
1.3 \\
2.6 \\
1.6 \\
0.9 \\
3.1 \\
2.0 \\
1.6\end{array}$ & 15.7 \\
\hline 745 & $\begin{array}{l}\text { MELIACEAE } \\
\text { Guarea aff. guidonia (L.) Sleumer }\end{array}$ & 6 & 3 & 1 & 2 & 605.4 & 0.9 & 1.1 & 2.0 & 4.0 & 3.1 \\
\hline 725 & $\begin{array}{l}\text { MENISPERMACEAE } \\
\text { Odontocarya zuliana Barneby }\end{array}$ & 1 & 1 & & & 1 & 0.2 & 0.0 & 0.5 & 0.7 & 1.1 \\
\hline $\begin{array}{l}795 \\
789 \\
800 \\
922 \\
785\end{array}$ & $\begin{array}{l}\text { MIMOSACEAE } \\
\text { Inga marginata Willd. } \\
\text { Inga sapindoides Willd. } \\
\text { Inga aff. tenuistipula Ducke } \\
\text { Inga aff. umbellifera (Vahl) Steud. ex DC. } \\
\text { Leucaena leucocephala (Lam.) de Wit }\end{array}$ & $\begin{array}{l}1 \\
2 \\
2 \\
1 \\
3\end{array}$ & $\begin{array}{l}1 \\
1\end{array}$ & $\begin{array}{l}1 \\
1 \\
1 \\
1\end{array}$ & 2 & $\begin{array}{r}3.4 \\
8.5 \\
333.9 \\
19.1 \\
466.4\end{array}$ & $\begin{array}{l}0.2 \\
0.3 \\
0.3 \\
0.2 \\
0.5\end{array}$ & $\begin{array}{l}0.0 \\
0.0 \\
0.6 \\
0.0 \\
0.8\end{array}$ & $\begin{array}{l}0.5 \\
1.0 \\
1.0 \\
0.5 \\
1.0\end{array}$ & $\begin{array}{l}0.7 \\
1.3 \\
1.9 \\
0.7 \\
2.3\end{array}$ & 8.2 \\
\hline $\begin{array}{l}749 \\
754 \\
767 \\
830 \\
827\end{array}$ & $\begin{array}{l}\text { MORACEAE } \\
\text { Brosimum alicastrum Sw. } \\
\text { Clarisia cf. biflora Ruíz \& Pav. } \\
\text { Ficus dugandii Standl. } \\
\text { Helicostylis tomentosa (Poepp. \& Endl.) J.F. Macbr. } \\
\text { Perebea aff. angustifolia (Poepp.\& Endl.) C.C. Berg }\end{array}$ & $\begin{array}{r}19 \\
6 \\
4 \\
1 \\
1\end{array}$ & $\begin{array}{r}13 \\
1\end{array}$ & $\begin{array}{l}6 \\
1 \\
1 \\
1\end{array}$ & $\begin{array}{l}4 \\
3\end{array}$ & $\begin{array}{r}198.3 \\
675.8 \\
3457.8 \\
7.2 \\
2.4\end{array}$ & $\begin{array}{l}3.0 \\
0.9 \\
0.6 \\
0.2 \\
0.2\end{array}$ & $\begin{array}{l}0.4 \\
1.2 \\
6.1 \\
0.0 \\
0.0\end{array}$ & $\begin{array}{l}1.9 \\
1.5 \\
1.5 \\
0.5 \\
0.5\end{array}$ & $\begin{array}{l}5.2 \\
3.6 \\
8.0 \\
0.7 \\
0.7\end{array}$ & 17.1 \\
\hline 798 & $\begin{array}{l}\text { MYRISTICACEAE } \\
\text { Virola sebifera Aubl. }\end{array}$ & 5 & & 3 & 2 & 703.0 & 0.8 & 1.2 & 1.5 & 4.0 & 3 \\
\hline 953 & $\begin{array}{l}\text { MYRSINACEAE } \\
\text { Myrsine pellucida (Ruíz \& Pav.) Spreng. }\end{array}$ & 3 & 1 & 1 & 1 & 499.7 & 0.5 & 0.9 & 0.5 & 1.8 & 2.3 \\
\hline 947 & $\begin{array}{l}\text { MYRTACEAE } \\
\text { Myrcia paivae O. Berg }\end{array}$ & 4 & 3 & 1 & & 11.3 & 0.6 & 0.0 & 1.0 & 1.6 & 1.6 \\
\hline 807 & $\begin{array}{l}\text { NYCTAGINACEAE } \\
\text { Neea divaricata Poepp. \& Endl. }\end{array}$ & 1 & & & 1 & 588.6 & 0.2 & 1.0 & 0.5 & 1.7 & 2.1 \\
\hline 978 & $\begin{array}{l}\text { OCHNACEAE } \\
\text { Rhytidanthera cf. regalis R.E. Schult. }\end{array}$ & 6 & 1 & 3 & 2 & 369.3 & 0.9 & 0.7 & 1.0 & 2.6 & 2.5 \\
\hline 805 & $\begin{array}{l}\text { OLACACEAE } \\
\text { Heisteria acuminata (Humb. \& Bonpl.) Engl. }\end{array}$ & 10 & 7 & 3 & & 80.0 & 2.0 & 0.1 & 1.0 & 2.7 & 2.6 \\
\hline
\end{tabular}




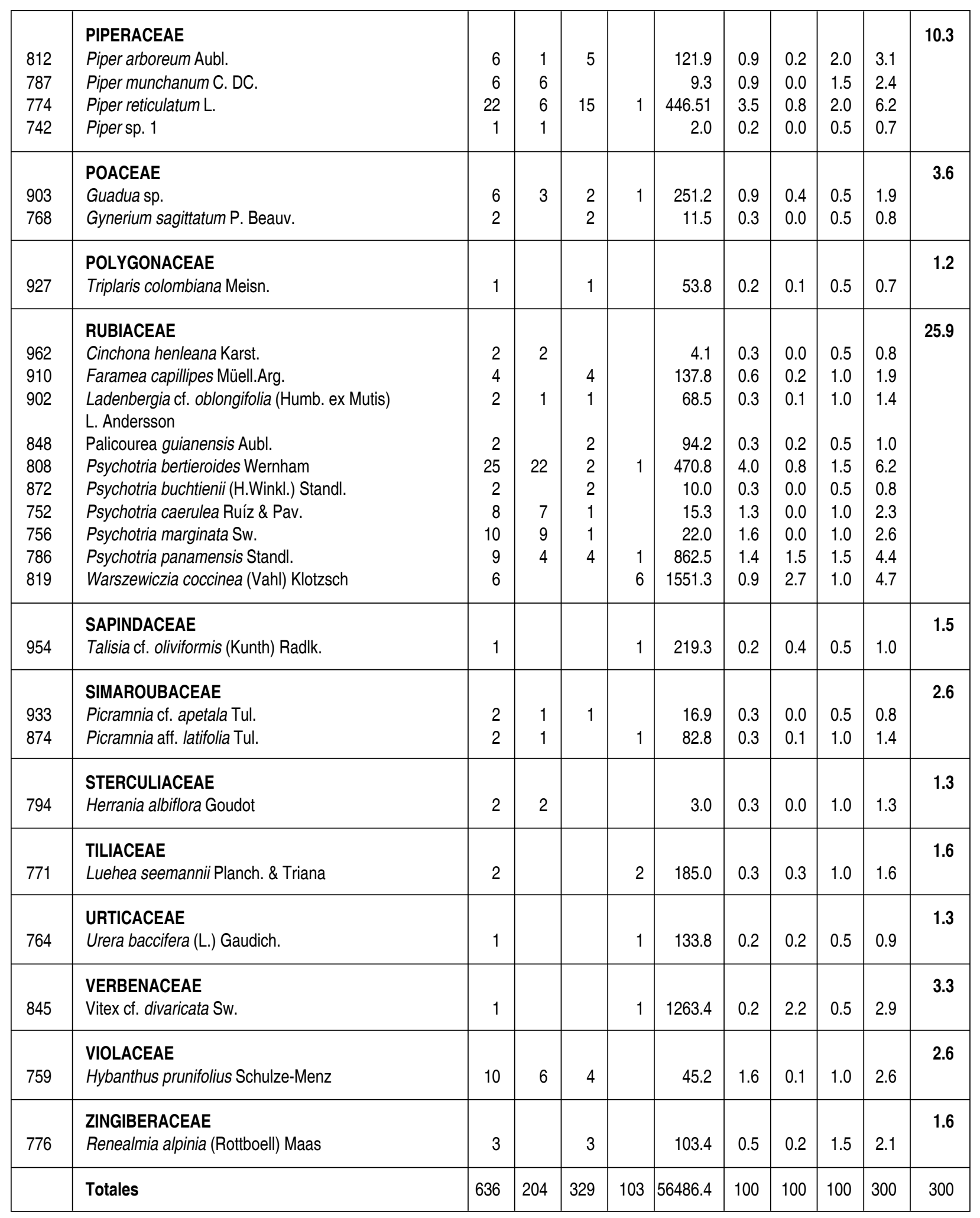

